\title{
Rakenteiden Mekaniikka -lehden 50 vuotisjuhlaseminaari 24-25.8.2017
}

\section{Tausta}

Rakenteiden Mekaniikan Seura Ry järjestää yhdessä Aalto-yliopiston, Jyväskylän yliopiston, Lappeenrannan teknillisen yliopiston, Oulun yliopiston ja Tampereen teknillisen yliopiston kanssa seminaarin juhlistamaan Rakenteiden Mekaniikka -lehden 50 vuotista historiaa. Lehti on perustettu 1968 ja siten vuosi 2017 on lehden 50. vuosikerta. Lehden verkkosivut http://rakenteidenmekaniikka.journal.fi/index.

Seminaarin aiheina ovat kiinteiden ja virtaavien aineiden teoreettinen ja laskennallinen sekä kokeellinen mekaniikka. Esimerkkeinä voidaan mainita virtausmekaniikka, rakenteen ja virtauksen vuorovaikutus, maamekaniikka, roottoridynamiikka, monikappaledynamiikka, murtumismekaniikka ja väsyminen, kantavien rakenteiden staattinen ja dynaaminen lujuusanalyysi, rakenteiden optimointi, dynaamisten systeemien optimaalinen säätö, numeeriset menetelmät, mekaniikan teolliset ja lääketieteelliset sovellutukset, jne.

Seminaarin tarkoituksena on koota yhteen tutkijoita, suunnittelijoita, opettajia sekä muita ammattilaisia, jotka työssään ovat tekemisissä mekaniikan, lujuusopin ja näihin liittyvien matemaattisten ongelmien kanssa.

Seminaari pidetään Vaasan yliopistolla.

\section{Ilmoittautuminen ja osallistumismaksu}

Osallistumismaksu on 400 euroa. Osallistumismaksuun sisältyy kahvit lounaat sekä juhlaillallinen ravintola Svenska Klubbenilla torstai iltana, sekä seminaarista tehty Rakenteiden Mekaniikka -lehden erikoisnumero 3/2017.

\section{Kutsutut esitelmöitsijät}

Claes Johnson, KTH, Structural mechanics of the atom

Anders Klarbring, Linköping, Robust structural topology optimization and game theory Pauli Jumppanen, Rakenteiden Mekaniikka -lehden perustaja ja ensimmäinen päätoimittaja, Historical aspects and milestones in the development of structural mechanics in Finland Aki Mikkola, LUT, SIM-platform - Sustainable product processes through real-time simulations

Hannu Tienhaara, Wärtsilä Finland Oy, Fifty years of structural mechanics and simulation in Wärtsilä

\section{Temaattiset sessiot}

Models, methods and applications of structural analysis/Rakenneanalyysin mallit, menetelmät ja sovellukset

Organized by Jarkko Niiranen, Antti Niemi, e-mail: jarkko.niiranen(at)aalto.fi, antti.niemi(at)oulu.fi

Multi scale modelling of materials/Materiaalien moniskaalamallinnus Organized by Anssi Laukkanen, Merja Sippola, e-mail: anssi.laukkanen(at)vtt.fi, merja.sippola(at)vtt.fi 
Mathematical methods in mechanics/Mekaniikan matemaattiset menetelmät

Organized by Heikki Orelma, e-mail: heikki.orelma(at)tut.fi

Computational methods in industry/Teollisuuden laskennalliset menetelmät

Organized by Tero Frondelius, e-mail: tero.frondelius(at)wartsila.com

Fretting fatigue modeling and measurements/Kitkaväsymisen mallintaminen ja mittaukset

Organized by Arto Lehtovaara, e-mail: arto.lehtovaara(at)tut.fi

Fatigue of materials and structures/Materiaalien ja rakenteiden väsyminen

Organized by Timo Björk, e-mail: timo.bjork(at)lut.fi, Reijo Kouhia, e-mail:

reijo.kouhia(at)tut.fi and Heikki Remes, e-mail: heikki.remes(at)aalto.fi

Seminaariin on lähetetty kutsuesitelmöitsijöiden lisäksi 66 esitystä. Suosituimpia aiheita ovat väsyminen, optimointi, numeeriset menetelmät ja suunnittelu. Tarkempi ohjelma on sivuilla III-IV.

\section{Majoittuminen}

Original Sokos Hotel Royal Vaasa, Hovioikeudenpuistikko 18 (65100 Vaasa) Standard Class on varattu kiintiö huoneita hintaan 94,90€ / yhden hengen huone / vrk tai 110,10€ / kahden hengen huone / vrk. Hinnat sisältävät runsaan buffetaamiaisen sekä sauna- ja allasosaston käytön. Kiintiöhinnat eivät oikeuta S-bonus pisteisiin tai etuihin.

Majoittujat varaavat itse huoneet kiintiöstä varauskoodia BRM50 käyttäen 7.8.2017 mennessä. Varaukset voi tehdä osoitteesta www.sokoshotels.fi tai hotellin myyntipalvelusta soittaen p. 062127267 tai sähköpostilla sales.royalvaasa@sokoshotels.fi

Cumulus Resort Tropiclandia, Lemmenpolku 3 (65170 Vaasa) tarjoaa huoneita hintaan: 89,00 EUR/ vrk / yhden hengen huone ja 99,00 EUR / vrk / kahden hengen huone Hintaan sis. majoitus, buffet-aamiainen ja hotellin iltasauna. Majoittujat varaavat itse huoneen www.restel.fi.

\section{Seminaarin verkkosivut}

Ajantasainen informaatio löytyy seminaarin verkkosivuilta http://rmseura.tkk.fi/rm50/. 


\section{Thursday 24.8.2017}

\section{Plenary I 8:40-10:10}

- Pauli Jumppanen; Historical aspects and milestones in the development of structural mechanics in Finland

- Claes Johnson; Structural mechanics of the atom

- Anders Klarbring; Robust structural topology optimization and game theory

\section{Session 1A: Fatigue 1 - analysis and modelling 10:40-12:20}

- Asko Kumpula, Joona Vaara, Anton Leppänen, Tero Frondelius; Nodular cast iron Onera fatigue model fitting

- Anton Leppänen, Asko Kumpula, Joona Vaara, Massimo Cattarinussi, Juho Könnö, Tero Frondelius; Thermomechanical fatigue analysis of cylinder head

- Miikka Väntänen, Joona Vaara, Jukka Aho, Jukka Kemppainen, Tero Frondelius; Bayesian sequential experimental design for fatigue tests

- Joona Vaara, Antti Mäntylä, Tero Frondelius; Brief review on high-cycle fatigue with focus on non-metallic inclusions and forming

- Joonas Jussila, Sami Holopainen, Terhi Kaarakka, Reijo Kouhia, Jari Mäkinen, Heikki Orelma, Niels Saabye Ottosen, Matti Ristinmaa, Timo Saksala, A new paradigm for fatigue analysis - evolution equation based continuum approach

Session 1B: Experimental mechanics 10:40-12:20

- Jukka Aho, Tero Frondelius; Analyzing 3 TB Field measurement data set

- Pasi Halla-aho, Antti Mäntylä, Tero Frondelius, Tommi Helander, Juha Hautala; Counterweight measurements device development

- Diana Burdiel, Carlo Pestelli, Moreno Almerigogna, Alan Pettirosso, Heikki Mikonaho, Teemu Kuivaniemi, Tero Frondelius; Matching simulated and measured eigen frequencies of medium speed diesel engine

- Tommi Helander; Pyörivien laitteiden etävalvonta/Remote condition monitoring of rotating machinery

- Seppo Moilanen, Ville Lehtinen; Suurikaliiperisten aseiden tuliputkien pyörrevirta-tarkastuksen ja ultraäänitarkastuksen mekanisoinnin kehitys ja toteutus

\section{Session 1C: Optimization 10:40-12:20}

- Timo Turkkila; Topology optimization of cast iron parts

- Joni Keski-Rahkonen; Probabilistic framework for product design optimization

- Evgeniya Kiseleva, Juho Könnö, Niclas Liljenfeldt, Teemu Kuivaniemi, Tero Frondelius; Topology optimisation of In-line engine turbocharger bracket

- Károly Jármai; Optimum design of welded structures

- Teemu Tiainen, Kristo Mela; Kaksivaiheinen menettely epälineaarisen diskreetin teräsrakenteiden optimointitehtävän ratkaisemiseksi

Plenary $2 \quad 13.20-14.30$

- Aki Mikkola; SIM-platform - Sustainable product processes through real-time simulations

- Hannu Tienhaara; Fifty years of structural mechanics and simulation in Wärtsilä

\section{Session 2A: Computational methods in industry 15:00-16:20}

- Antti Korpela, Marko Jokinen, Teemu Kuivaniemi, Tero Frondelius; W4L20 VEBIC genset dynamics baseframe design

- Jussi Göös, Antti Mäntylä, Anton Leppänen, Tero Frondelius; Large bore connecting rod simulations

- Ilkka Väisänen, Antti Mäntylä, Antti Korpela, Tero Frondelius; Medium speed engine crankshaft analysis

- Teemu Kuivaniemi, Antti Mäntylä, Ilkka Väisänen, Antti Korpela, Tero Frondelius; Dynamic gear wheel simulations using multibody dynamics

\section{Session 2B: Fretting fatigue modeling and measurements 15:00-16:20}

- Jouko Hintikka, Janne Juoksukangas, Arto Lehtovaara, Tero Frondelius, Antti Mäntylä; Non-idealities in fretting contacts

- Antti Mäntylä, Jussi Göös, Anton Leppänen, Tero Frondelius; Large bore engine connecting rod fretting analysis

- Jouko Hintikka, Arto Lehtovaara, Tero Frondelius, Antti Mäntylä; Tangential traction instability in fretting contact below fully developed friction load

- Juho Niva; Fretting-säröjen etenemisen tutkiminen murtumismekaniikan avulla

Session 2C: Models, methods and applications of structural analysis 1 15:00-16:20

- Jouni Freund; Two-scale Reissner-Mindlin plate model

- Jarkko Niiranen, Sergei Khakalo, Viacheslav Balobanov; Isogeometric finite element analysis of mode I cracks within strain gradient elasticity

- Markku Raiskila, Joona Tuikka; IFC- tiedostomuodon käyttö FEM- mallinnuksessa

- Timo Björk, Niko Tuominen, Antti Ahola; X-liitoksen vinoutumisnurjahdus 


\section{Friday 25.8.2017}

Session 3A: Models, methods and applications of structural analysis 2 8:40-10:20

- Tero Frondelius, Jukka Aho; JuliaFEM - open source solver for both industrial and academic usage

- Jarno Jokinen, Mikko Kanerva; Sensitivity analysis for simulated testing of composites: mapping via Isight and Abaqus

- Mika Malinen; Shell finite elements: implementation with Elmer software

- Marja Rapo, Jukka Aho, Tero Frondelius; Natural frequency calculations with JuliaFEM

- Timo Saksala; Numerical modelling of rock materials with polygonal finite elements

\section{Session 3B: Mathematical methods in mechanics 8:40-10:20}

- Tom Gustafsson, Rolf Stenberg, Juha Videman; A posteriori analysis of classical plate elements

- Mikael Nyberg, Antti Mäntylä, Tero Frondelius; Explosion simulation of pressurized components

- Juhani Taipale; Rakennejärjestelmän toisen asteen tekijöiden luotettavuusteoreettinen yhteisvaikutusanalyysi

- Tuomo Poutanen, Rakenteiden mekaniikka Eurokoodeissa

\section{Session 3C: Fatigue 2 - structural and testing 8:40-10:20}

- Ilkka Valkonen, Antti Valkonen: Tuotantokäyttöön soveltuva edullinen menetelmä hitsin juuren puolen väsymiseliniän arvioimiseksi

- $\quad$ Lassi Keränen; Kyntöauran rakenteellinen kehittäminen mitatun kuormitusaineiston perusteella

- Antti Ahola, Heli Mettänen, Timo Björk; Kuormitustavan ja symmetrisyyden vaikutus kuormaa kantamattomien hitsausliitosten väsymiseen - tehollisen lovijännityksen ja murtumismekaniikan menetelmien vertailu

- Jussi Korhonen, Antti Mäntylä. Juho Könnö, Tero Frondelius; Full scale fatigue testing of crankshaft

- Jussi Korhonen, Juha Kuoppala, Miikka Väntänen, Joona Vaara, Mikko Turunen, Panu Kämäräinen, Jarkko Laine, Aulis Silvonen, Tero Frondelius: QT steel high cycle fatigue testing with ultrasonic

\section{Session 4A: Multi scale modelling of materials 10:50-12:10}

- Tomas Their, Luc St-Pierre, Stiffness and strength of a semi-regular lattice

- Stefania Fortino, Merja Sippola, Tom Andersson, Kirsi Immonen, Arttu Miettinen, Petr Hradil; X-ray micro-tomography based FEM modelling of hygroexpansion in PLA composites reinforced with birch pulp fibers

- Merja Sippola, Anssi Laukkanen, Tom Andersson, Matti Lindroos; Microstructural modelling of materials

- Anssi Laukkanen, Matti Lindroos, Tom Andersson, Tuukka Verho, Tatu Pinomaa; Micromechanical modeling of failure behavior of metallic materials

Session 4B: Dynamics 10:50-12:10

- $\quad$ Liang Bai, Teemu Kuivaniemi, Pasi Halla-aho, Tero Frondelius; Elastohydrodynamic simulation of the slider bearing

- Matti Lindstedt; Combined mesh and frame motion for fast transient CFD simulation of rotating machines

- Tapani Kukkola, Pentti Varpasuo; Fortum contribution to the Iris phase 3 benchmark study

- Pekka Salmenperä, Robert Hildebrand; Ratapenkereen värähtelyriski nopeuden noustessa

\section{Session 4C: Structural mechanics 1 10:50-12:10}

- Hadi Fathipour Azar, Timo Saksala, Sayed Esmaiel Jalali; Artificial neural networks models for rate of penetration prediction in rock drilling

- Antti-Jussi Vuotikka, Mikael Nyberg, Heikki Karhinen and Tero Frondelius; Contact sealing simulation of high pressured diesel injector

- Antti H. Niemi, Jani Koskela, Filip Fedorik; Evaluation of some harmonic load models for the vibration analysis of footbridges.

\section{Session 5A: Design approaches 13:00-14:40}

- Kai Katajamäki; Automatic simulation platform to support product design and optimization

- $\quad$ Erin Komi, Petteri Kokkonen; Design approaches for additive manufactured components, with a focus on selective laser melting

- Massimo Cattarinussi, Anton Leppänen, Juho Könnö, Tero Frondelius; Cylinder head design of experiment by using the Wärtsilä Digital Design Platform

- Johannes Heilala, Teemu Kuivaniemi, Juho Könno, Tero Frondelius; Calculation tool for dynamics of generator set common baseframe

- J. Könnö, H. Tienhaara, T. Frondelius; Wärtsilä digital design platform

Session 5B: Electromechanics 13:00-14:40

- Anouar Belahcen; Stress Effect on magnetostriction measurement in electrical sheets under rotational magnetization

- Janne Keränen, Pavel Ponomarev, Sabin Sathyan, Juhani Kataja, Anouar Belahcen; Magneto-structural simulation of an induction motor start-up using nodal magnetic forces in Elmer

- Juan Chowdhury, Gaurav Kumar, Karuna Kalita, Kari Tammi, Sashindra K. Kakoty; A brief overview on linear switched reluctance Motor

- Deepak Singh, Kati Mökkönen, Jarmo Poutala, Paavo Rasilo, Anouar Belahcen, Reijo Kouhia; Preliminary results on the effect of plastic deformation on magnetic properties

- Siavash Danaee, Reijo Kouhia, Paavo Rasilo, Anouar Belahcen, Deepak Singh, Matti Ristinmaa; A model for magnetoelastic behaviour

\section{Session 5C: Structural mechanics 2 13:00-14:40}

- Marsel Garifullin, Jarmo Havula, Sami Pajunen, Kristo Mela, Markku Heinisuo; Initial in-plane rotational stiffness of welded rectangular hollow section $\mathrm{T}$ joints with axial force at the main member

- Mohammad Dabiri, Mehran Ghafouri, Timo Björk; Estimation of stress concentration factors in butt and T-welded joints using artificial neural network-based models

- $\quad$ Teemu Peltoniemi; Ultralujan teräksen hitsiliitoksen äärikestävyys

- Pentti Varpasuo, Timo Kirkkomäki; Imatran vesivoimalaitos, koneisto nro 7, generaattorin perustusten korjaus 